\title{
Research on the crosslinking mechanism of polyacrylamide/resol using molecular simulation and X-ray photoelectron spectroscopy
}

\author{
Tao Ni, Guang-Su Huang, Jing Zheng, Pin Gao and Meng-Meng Chen
}

Resol crosslinked polyacrylamide (PAM) hydrogel can be used as the chemical flooding agent in enhanced oil recovery because of its excellent temperature- and salt-resistant properties. It is known that crosslinking reactions, including ortho-ortho, orthopara and para-para polycondensation of resols and ortho and para polycondensation between PAM and resol, are involved in the researched system, but the extent and activity of the reactions are still not clear. In this study, these crosslinking reactions were all investigated by a combination of molecular simulation and X-ray photoelectron spectroscopy (XPS) techniques. When propionamide was investigated as the model compound of PAM, the Gibbs-free energy and energy barrier of the reactions were calculated by density-functional theory. The atomistic model of the crosslinked PAM network was constructed by molecular mechanics and molecular dynamics, and the density of crosslinked PAM and the amount of active crosslinking sites were obtained. The results show that these reactions occurred spontaneously at $353 \mathrm{~K}$ because of thermodynamics. The ortho condensation between PAM and resol is the main kinetic reaction, and the reacted amidocyanogen reached $60 \%$ of the total amount, which is consistent with the XPS analysis.

Polymer Journal (2010) 42, 357-362; doi:10.1038/pj.2010.10; published online 10 March 2010

Keywords: crosslinking mechanism; molecular simulation; polyacrylamide hydrogel; trihydroxymethylphenol

\section{INTRODUCTION}

A polymer gel consists of an elastic crosslinked network and a fluid filling the interstitial space of the network. ${ }^{1-2}$ Ordinary polymer gels contain polystyrene gel, poly(dimethyl)siloxane gel, polyacrylamide (PAM) gel and other materials. Among the gels, PAM hydrogel has been widely used in agriculture, water cleaning and medical fields, owing to its features of absorbing and retaining water, ${ }^{3}$ separating and preconcentrating some heavy-metal ions, ${ }^{4}$ loading drugs and releasing drugs. $^{5}$ Its recent successful applications for reducing water have aroused interest within the oil production industry, ${ }^{6-8}$ especially in enhanced oil recovery. Until now, two principal types of crosslinkers, metal ions [Al (III), Cr (III) $\mathrm{Zr}$ (IV) $]^{9-10}$ and organic systems (particularly phenol-formaldehyde or resol), have been used in oil production with PAM. Research ${ }^{11-12}$ has shown that metal-ion crosslinkers seem to be suitable only for low temperature applications because of their fast reaction, precipitation and retention in porous media at high temperatures. On the contrary, organic crosslinkers can gel slowly and bear relatively high temperatures, prompting them to be candidates for chemical flooding in enhanced oil recovery. The different types of crosslinkers lead to discrepancies in rheological properties, salt resistance and temperature resistance; moreover, crosslinking density and junction size also have an effect on these properties, ${ }^{13-15}$ hence comprehension of the structure-function relation has become increasingly important. However, it is difficult to characterize the structure and composition of the crosslinked products with poor solubility and meltability, hence the crosslinked structure and mechanism are not clearly understood. At present, the crosslinking mechanism of the system has not been fully documented, ${ }^{16}$ retarding the development of resol/partially hydrolyzed PAM and the improvement in enhanced oil recovery.

Molecular simulation can simulate the structure and property of the system on the atomic-molecular level, and thus it could solve some difficulties encountered in the reaction process. In this study, molecular mechanics, molecular dynamics (MD) and density functional theory with high precision and high efficiency were applied to model the crosslinking reaction mechanism of PAM/resol. With the aid of $\mathrm{X}$-ray photoelectron spectroscopy (XPS), a crosslinking reaction mechanism is proposed.

\section{EXPERIMENTAL PROCEDURE}

Preparation of PAM hydrogel

PAM was synthesized by free-radical polymerization of acrylamide in a water solution at $303 \mathrm{~K}$ using potassium persulfate and sodium bisulfite as initiators. The obtained PAM was dissolved in water and mixed with 2,4,6-trihydrox- 
<smiles>OCc1cc(CO)c(O)c(CO)c1</smiles><smiles>CCCC(CC(C)(C)C)C(N)=O</smiles>

Figure 1 Structure of 2,4,6-trihydroxymethylphenol (a) and polyacrylamide (b).

ymethylphenol (THMP) (prepared according to literature ${ }^{17}$ ) in a ratio of 1:1 (weight ratio). The solution was charged into a scaled glass ampoule and degassed with nitrogen to remove air, then aged at $353 \mathrm{~K}$. A week later, the crosslinked resultant was dried in a vacuum oven, extracted with ethanol for $72 \mathrm{~h}$ and finally dried to afford the product.

\section{XPS characterization}

XPS measurements were performed on an XSAM800 X-ray photoelectron spectrometer (KRATOS, Dublin, Ireland) under $5 \times 10^{-7} \mathrm{~Pa}$ using $\mathrm{MgK} \alpha$ as a monochromatic X-ray source. The binding energy was calibrated by C1s at $284.8 \mathrm{eV}$.

\section{Simulations of reaction thermodynamics and kinetics}

As indicated in Figure 1, the reactive groups of PAM and resol are amidocyanogen and hydroxymethyl in crosslinking reactions, respectively. The reactivity of the hydroxymethyl groups in phenol is considered to be different, hence the following five reactions may occur in the system: ortho-ortho, ortho-para and para-para polycondensation of resols and ortho and para polycondensation between PAM and resol. These reactions were simulated using the density functional theory technique and propanamide (PA) was regarded as the model compound of PAM. The molecules of the corresponding reactants and products were prepared as reactant files and product files, in which their initial structures were generated through Energy and Geometry Optimization by the $\mathrm{DMol}^{3}$ program, ${ }^{18-19}$ which is available in Accelrys' Materials Studio (San Diego, CA, USA). GGA and BP were used as the function, and the frequency property, medium SCF tolerance and smearing of orbital occupancies were used. The relationships between reactant files and product files were connected by implementation of Reaction Preview, and the resulting files were named as trajectory files. A transition state (TS) search was used to find possible TS according to the activated trajectory files; the minimum energy of saddle points was considered to be the final TS.

\section{Construction of crosslinking net}

The structure of the crosslinked polymer network was established by the Amorphous Cell module, and periodic boundary conditions were set at the same time, resulting in a network composed of two PAM chains $(\mathrm{Dn}=20)$ and 16 THMP molecules. The energy-minimized three-dimensional periodic molecular systems were established by molecular mechanics and MD optimization in the COMPASS force field using Discovery from Accelrys. The process of MD was carried out by an annealing technique consisting of $1000 \mathrm{ps} \mathrm{MD}$ at $300 \mathrm{~K}$, another $1000 \mathrm{ps} \mathrm{MD}$ at $600 \mathrm{~K}$ then $1000 \mathrm{ps} \mathrm{MD}$ at $353 \mathrm{~K}$, using the constant volume and temperature ensemble (NVT). The resulting physical mix of PAM and THMP has been analyzed to identify the reactive sites in close proximity. The sites with interatomic distances within $6 \AA$ were considered to be the participants of the crosslinking reactions according to several trials of various distances. ${ }^{20}$ The reactive sites were chemically linked together with the release of water molecules from the system. The resultant molecular systems have been equilibrated to remove the strain imposed by the bond breaking/creating process using the MD (NVT) process for $200 \mathrm{ps}$ at $353 \mathrm{~K}$. By allowing all the probable reactive sites to react by repeating the above operations, the crosslinked net was finally built up and the density of crosslink production was given.

\section{RESULTS AND DISCUSSION}

\section{Thermodynamic and kinetic analysis of reactions}

A thermodynamic property, founded by Josiah Willard Gibbs in 1876, is usually applied to predict whether a process occurs spontaneously at constant pressure and temperature. Gibbs-free energy $(G)$ is defined as $G=H-T S$, where $H, T$ and $S$ are enthalpy, absolute temperature and
Table 1 Thermodynamic parameters obtained by $\mathrm{DMol}^{3}$

\begin{tabular}{|c|c|c|c|c|}
\hline Reaction system & $\mathrm{E}_{\text {electron }}(\mathrm{Ha})$ & $\begin{array}{c}\mathrm{G}^{\prime}\left(\mathrm{kcal} \mathrm{mol}^{-1},\right. \\
353 \mathrm{~K})\end{array}$ & $\begin{array}{c}\Delta \mathrm{G}\left(\mathrm{kcal} \mathrm{mol}^{-1}\right. \\
353 \mathrm{~K})\end{array}$ & $\mathrm{K}_{\mathrm{c}}$ \\
\hline \multicolumn{5}{|c|}{ o-Condensation of THMPS } \\
\hline Reactants & -1302.4085567 & 205.957 & -2.26 & $2.5 \times 10^{1}$ \\
\hline Products & -1302.3987633 & 197.548 & & \\
\hline \multicolumn{5}{|c|}{ o/p-Condensation of THMPs } \\
\hline Reactants & -1302.3869906 & 201.384 & -8.77 & $2.8 \times 10^{5}$ \\
\hline Products & -1302.4001329 & 200.861 & & \\
\hline \multicolumn{5}{|c|}{ p-Condensation of THMPs } \\
\hline Reactants & -1302.3812325 & 202.819 & -11.65 & $1.7 \times 10^{7}$ \\
\hline Products & -1302.4026801 & 204.626 & & \\
\hline \multicolumn{5}{|c|}{ o-Condensation of PA and THMP } \\
\hline Reactants & -899.7931643 & 150.260 & -0.36 & 1.67 \\
\hline Products & -899.7876390 & 146.430 & & \\
\hline \multicolumn{5}{|c|}{ p-Condensation of PA and THMP } \\
\hline Reactants & -899.7732990 & 147.294 & -13.98 & $4.8 \times 10^{8}$ \\
\hline Products & -899.7965104 & 147.878 & & \\
\hline
\end{tabular}

Abbreviations: o represents the ortho-position and $p$ represents the para-position; PA, propionamide; THMP, trihydroxymethylphenol.

Note: 1 Hartree $=627.51 \mathrm{kcal} \mathrm{mol}^{-1}$.

entropy, respectively. The change in Gibbs-free energy $(\Delta G)$ is negative for all spontaneous processes and zero for processes at equilibrium. $\Delta G$ is a state function, meaning that it only depends on the states of the reactant and the product. In this study, the condensation reaction can be expressed as:

$$
A+B \leftrightharpoons C+D
$$

The equilibrium constant of the reaction is given by

$$
\begin{aligned}
& K c=\exp (-\Delta G / R T) \\
& \Delta G_{\text {reaction }}=G_{\text {products }}-G_{\text {reactants }}
\end{aligned}
$$

Thermodynamic data can be calculated by quantum chemistry calculations. ${ }^{21} G$ can be written as

$$
\begin{aligned}
& G=E_{\text {electron }}+Z V P E+H_{\mathrm{T}}-H_{0}-T S \\
& G^{\prime}=Z V P E+H_{\mathrm{T}}-H_{0}-T S \\
& H_{\mathrm{T}}-H_{0}=\left(E_{\mathrm{vib}}+E_{\text {transt }}+E_{\text {rot }}\right)+R T
\end{aligned}
$$

where $E_{\text {electron }}$ is the total electronic energy, which can be obtained by geometric optimization, $R$ is the ideal gas constant $\left(8.314 \mathrm{~J} \mathrm{~mol}^{-1} \mathrm{~K}^{-1}\right)$, ZVPE is the zero-point vibrational energy, $H_{\mathrm{T}}-H_{0}$ is the enthalpy correction and $G^{\prime}$ is the finite temperature-corrected value for $G$, which can be calculated by vibrational frequency analysis on the basis of statistical mechanics.

Table 1 lists the thermodynamic parameters of $E_{\text {electron }}$ and $G^{\prime}$ of the reactants and products, along with $\Delta G$ and $K_{\mathrm{c}}$ of the reactions at certain temperatures. $K_{\mathrm{c}}$ and $\Delta G$ were calculated by Equations (2) and (3) on the basis of $E_{\text {electron }}$ and $G^{\prime}$. Obviously, $\Delta G$ of the five reactions is negative, hence these reactions occur spontaneously at $353 \mathrm{~K}$ on the basis of thermodynamics. The order of low-high $\Delta G$ is as follows: para PA/THMP condensation, para THMP/THMP condensation, ortho-para THMP/THMP condensation, ortho THMP/THMP condensation and ortho PA/THMP condensation. Conversely, the $K c$ of para PA/THMP condensation reached $4.8 \times 10^{8}$, the maximum among the above reactions, and the $K c$ of ortho PA/THMP only reached 1.67, the 
minimum among the reactions. From the results, it can be concluded that the probability of para condensation is higher than that of ortho condensation. The conclusion can also be explained by the stability of the structure of the final products. The structures of the para reaction products are stretched, and the distribution of space is symmetrical. By contrast, the $E_{\text {electron }}$ of para condensation is lower than that of ortho condensation. As a result, the structure and energy provide the products with good stability.

Thermodynamics addressed the feasibility and extent of the reactions, but kinetics must be considered at the same time. For a reaction to take place, the reactant molecules must have enough energy to overcome the energy barrier. The lower the reaction energy barrier, the faster the reaction. The energy barrier was calculated by a TS search.

TS structure can be searched by several methods, of which linear synchronous transfer (LST) and quadratic synchronous transfer (QST), together with conjugate gradient (CG), are considered to be

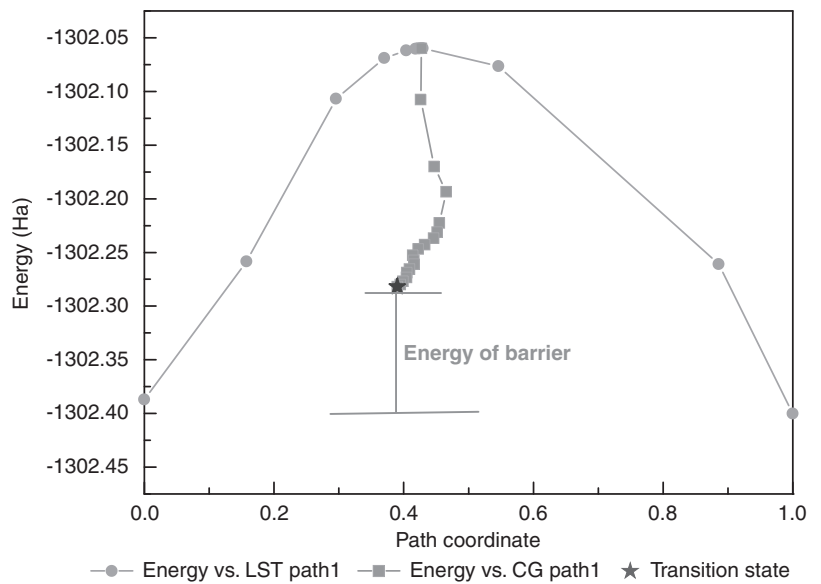

Figure 2 Transition state search of $\mathrm{o} / \mathrm{p}$-condensation of trihydroxymethylphenols. the best methods. Figure 2 displays the results of a TS search of orthopara self-condensation of THMP molecules; the TS ( $\star$ ) has been found by linear synchronous transfer and conjugate gradient. Figure 3 displays the results of the TS of ortho condensation of PA/THMP, the TS of which has also been obtained by linear synchronous transfer, quadratic synchronous transfer and conjugate gradient. The search process of PA/THMP condensation was more complicated than that of THMP/THMP condensation, which was determined from the degree of convergence; hence the search process should have no effect on the calculated energy barrier. The energy barrier based on the products is indicated in Figures 2 and 3. It can be clearly seen that the energy barrier of ortho condensation of PA/THMP is lower than that of the ortho-para condensation of the THMP molecule.

To simplify the discussion, the process of the TS search of the other reactions is not shown, and the results of only the TS search are shown in Table 2. Among the THMP/THMP systems, the energies of the TSs decrease in the sequence of para, ortho and ortho-para condensation. The TS energy of ortho condensation is lower than that of para condensation for the PA/THMP system. As the energy of the TS cannot determine the reaction activity unless the relative reactants (or products) are the same, the reaction energy barrier must be considered for these five different reactions. In this study, the order of energy barriers is ortho PA/THMP condensation $<$ ortho-para THMP/

Table 2 The energy obtained by transition state search

\begin{tabular}{lcc} 
Reaction types & Energy of TS (Ha) Energy of barrier $\left(\mathrm{kcal} \mathrm{mol}^{-1}\right)$ \\
\hline o-condensation of THMPs & -1302.2811194 & 79.98 \\
o/p-condensation of THMPs & -1302.2818610 & 65.99 \\
p-condensation of THMPs & -1302.1241315 & 161.35 \\
o-condensation of PA and THMPs & -899.7685780 & 15.44 \\
p-condensation of PA and THMPs & -899.6222733 & 94.78
\end{tabular}

Abbreviations: o represents the ortho-position and $p$ represents the para-position; PA, propionamide; TS, transition state; THMP, trihydroxymethylphenol.

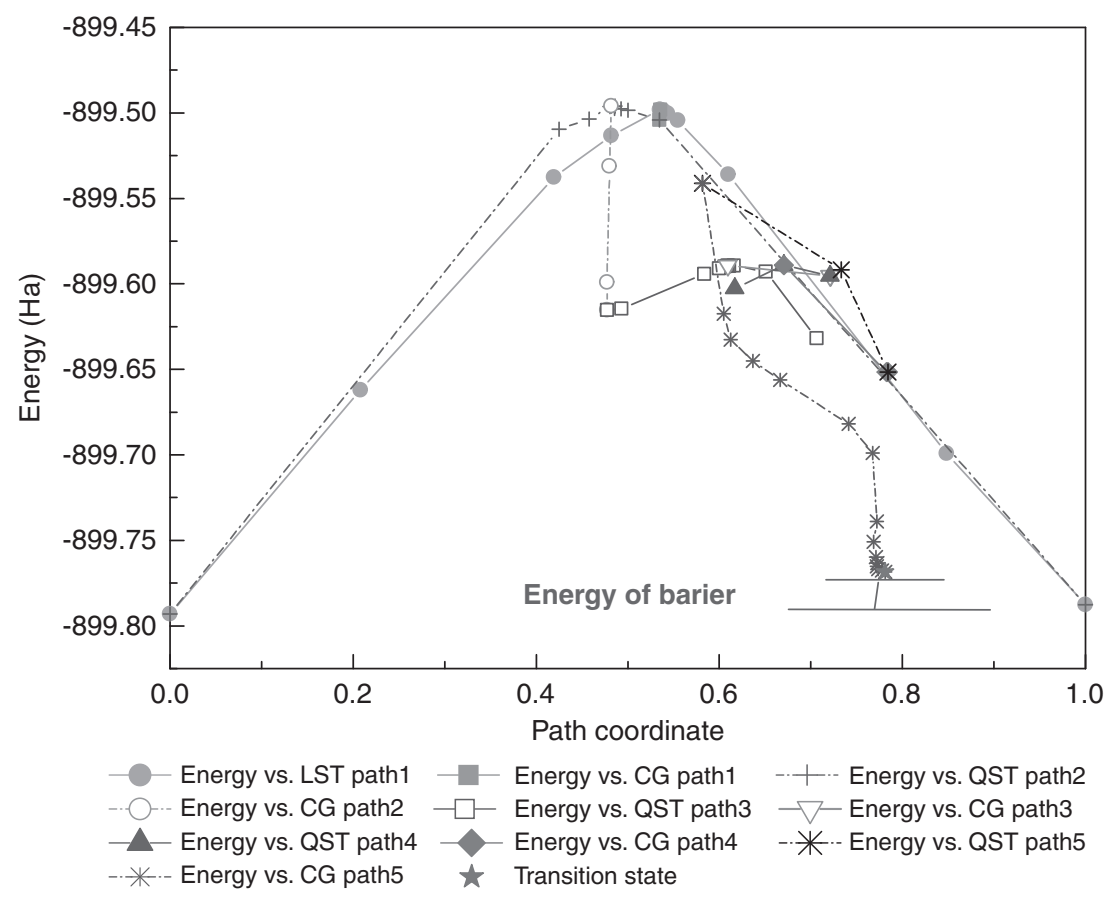

Figure 3 Transition state search of o-condensation of propionamide and trihydroxymethylphenol. 


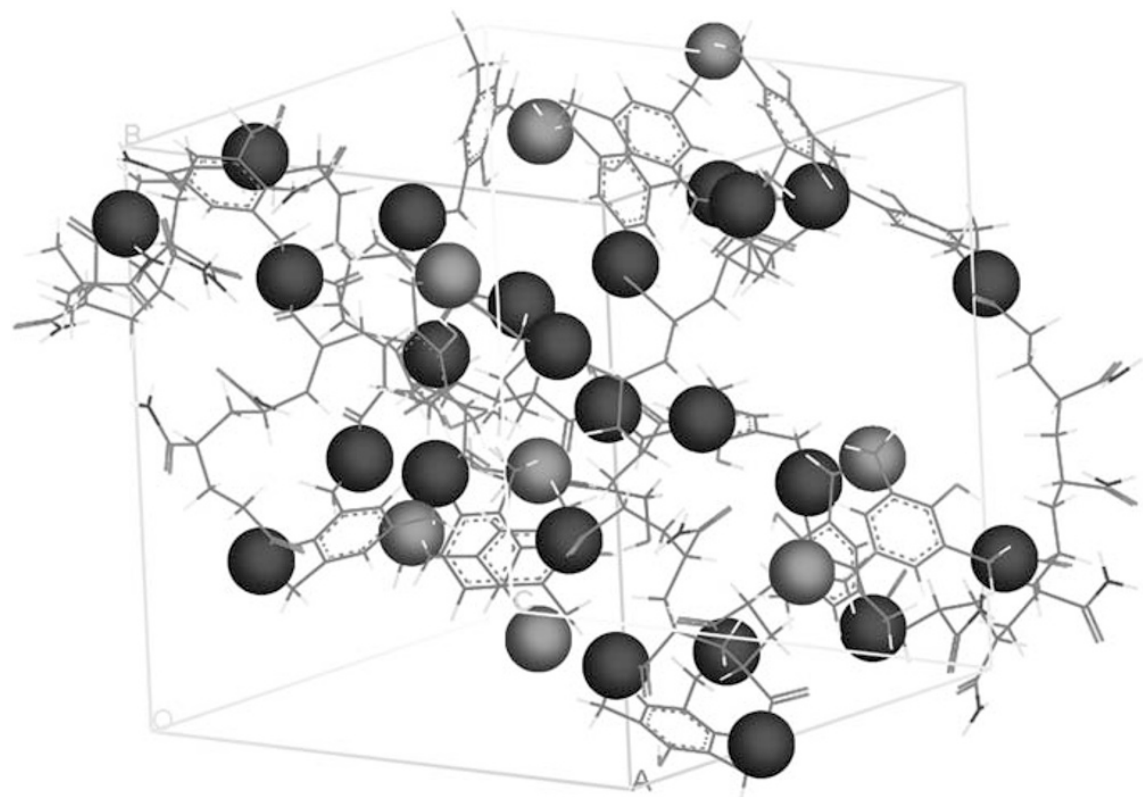

Figure 4 Crosslinked net structure of polyacrylamide/trihydroxymethylphenol. A full color version of this figure is available at the Polymer Journal online.

THMP condensation $<$ ortho THMP/THMP condensation $<$ para PA/THMP condensation $<$ para THMP/THMP condensation. By contrast, according to the Arrhenius equation, the rate constant $(K)$ can be obtained as:

$$
K=A \exp (-E a / R T)
$$

where $A$ is a constant and $E a$ is the energy barrier for the reaction. Being an exponent, the energy barrier affects the rate constant largely at the same temperature. The energy barrier of the ortho condensation reaction for PA/THMP is only $15.44 \mathrm{kcal} \mathrm{mol}^{-1}$, a relatively low energy for a reaction; hence this reaction can easily overcome the energy barrier and proceed relatively fast. However, the energy barrier of ortho-para PA/THMP condensation is $65.99 \mathrm{kcal} \mathrm{mol}^{-1}$, higher than that of the former, hence the reaction rate is very slow. For the other reactions with a higher energy barrier (more than $65.59 \mathrm{kcal} \mathrm{mol}^{-1}$ ), the reaction rate is much slower. On the basis of the above analysis, we are sure that the ortho condensation of PA/THMP is the most active and predominant reaction in the system.

When this conclusion is applied to the PAM/resol system, we predict that the polycondensation between resol and PAM is the main reaction. Because of their relative ratio of 2:16 (molar ratio), which can be transformed into the ratio (40:48) of the amidocyanogen and hydroxymethyl groups, the reacted amide can reach about $60 \%$ of the total amide according to $K c(1.67)$. The moderate conversion is a benefit for weak gel preparation of PAM/resol, as is reasonable and consistent with the experiment.

\section{Crosslinking reaction}

According to the active sequence achieved by the above simulations, the crosslinked polymer network and the average density of the crosslinking products could be obtained. The simulated periodic cell is shown in Figure 4.

In Figure 4, red balls represent oxygen atoms produced by condensation of resols and blue balls represent nitrogen atoms formed by condensation of PAM and resol. To analyze the crosslinked structure, the density of crosslinking products and the type of crosslinking points are presented in Table 3. The density of PAM is
Table 3 The results of the simulation for crosslinking gel

\begin{tabular}{lccc}
\hline System & $\begin{array}{c}\text { Density } \\
\left(\mathrm{gcm}^{-3}\right)\end{array}$ & $\begin{array}{c}\text { Types of } \\
\text { crosslink points }\end{array}$ & $\begin{array}{c}\text { The number of } \\
\text { crosslink point }\end{array}$ \\
\hline Polyacrylamide & 1.30 & - & - \\
Polyacrylamide crosslinked & 1.16 & P2408 & 32 \\
\hline
\end{tabular}

Note: $\mathrm{P}$ represents the crosslinked point between $-\mathrm{OH}$ and $-\mathrm{CONH}_{2}, \mathrm{O}$ represents the crosslinked point between $-\mathrm{OH}$ and $-\mathrm{OH}$.

$1.30 \mathrm{~g} \mathrm{~cm}^{-3}$ and the density decreases to $1.16 \mathrm{~g} \mathrm{~cm}^{-3}$ after crosslinking, which is consistent with the density tests. The drop in density after crosslinking can be explained by the resol molecules having supported a number of networks among the PAM molecules, expanding the product volume. In addition, the number of the crosslinked points of PAM/THMP (24) is greater than that of THMP/THMP (8), and the reacted amidocyanogen occupied $60 \%$ of the total amidocyanogen in the crosslinking process, which further confirmed that the condensation reaction between PAM and resol is the dominant reaction in the system.

XPS analysis of the crosslinking products

Crosslinked products are hard, insoluble and nonmelting, which makes it difficult to characterize their structures and compositions. XPS can analyze the chemical elemental composition and chemical state of solid samples, with an absolute sensitivity that can reach as high as $10^{-18}$; hence XPS is a good characterization tool for crosslinked products.

The N1s regions of uncrosslinked and crosslinked PAM were superposed (Figure 5), and it can be seen that the N1s region of crosslinked PAM has a higher binding energy compared with that of uncrosslinked PAM. The detailed chemical information on the surface of crosslinked PAM was obtained by fitting XPS spectra with the standard peak, then fitting the N1s spectra, as shown in Figure 6. The N1s spectra of crosslinked PAM gave two main peaks with binding energies at 399.2 and $400.0 \mathrm{eV}$, which correspond to unreacted amidocyanogen and reacted amidocyanogen, ${ }^{22-23}$ respectively. The area integral of the peak at $399.2 \mathrm{eV}(109.33)$ was lower than that at 


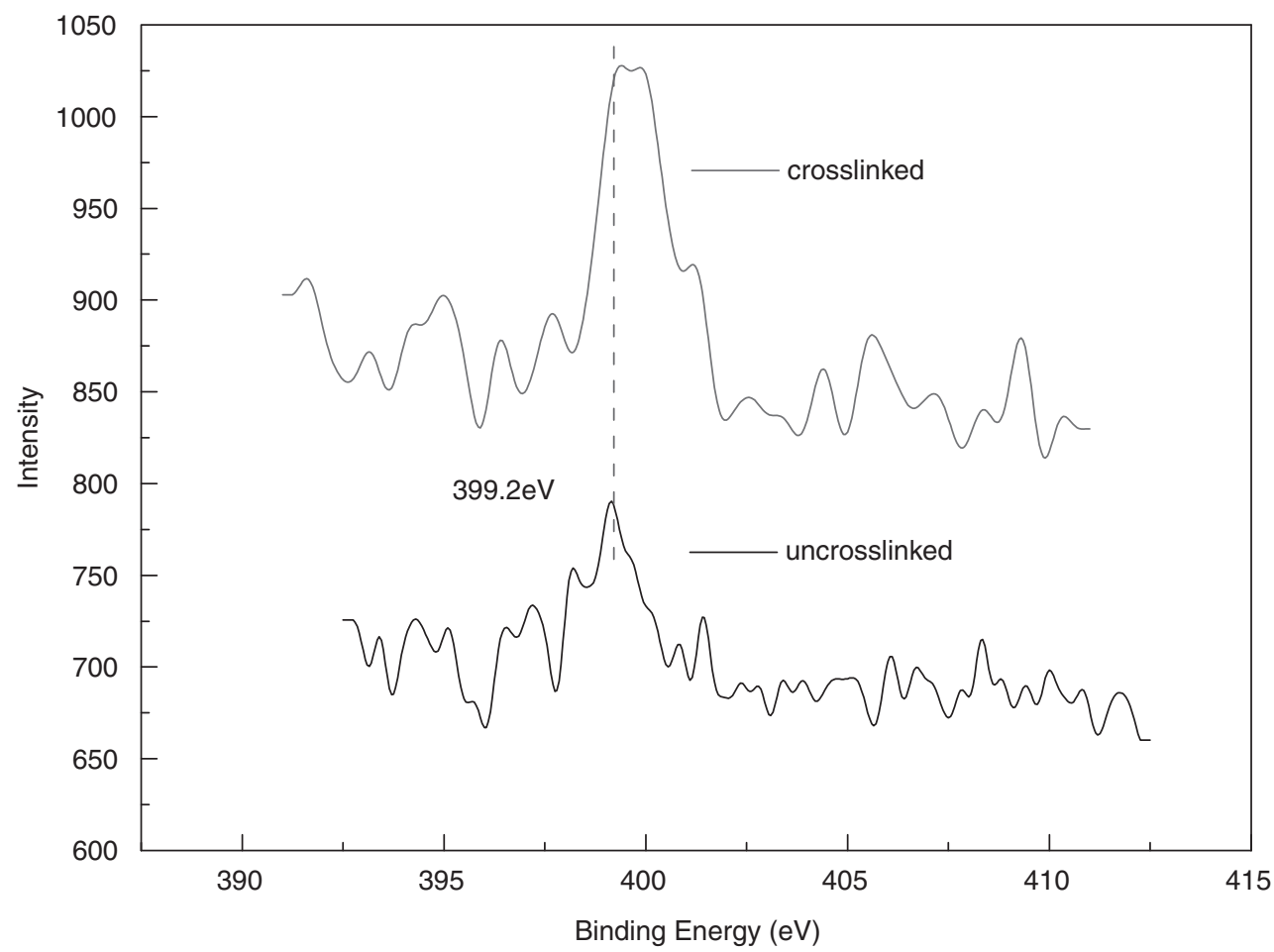

Figure $5 \mathrm{~N}_{1 \mathrm{~s}}$ electron spectra of uncrosslinked and crosslinked polyacrylamide.

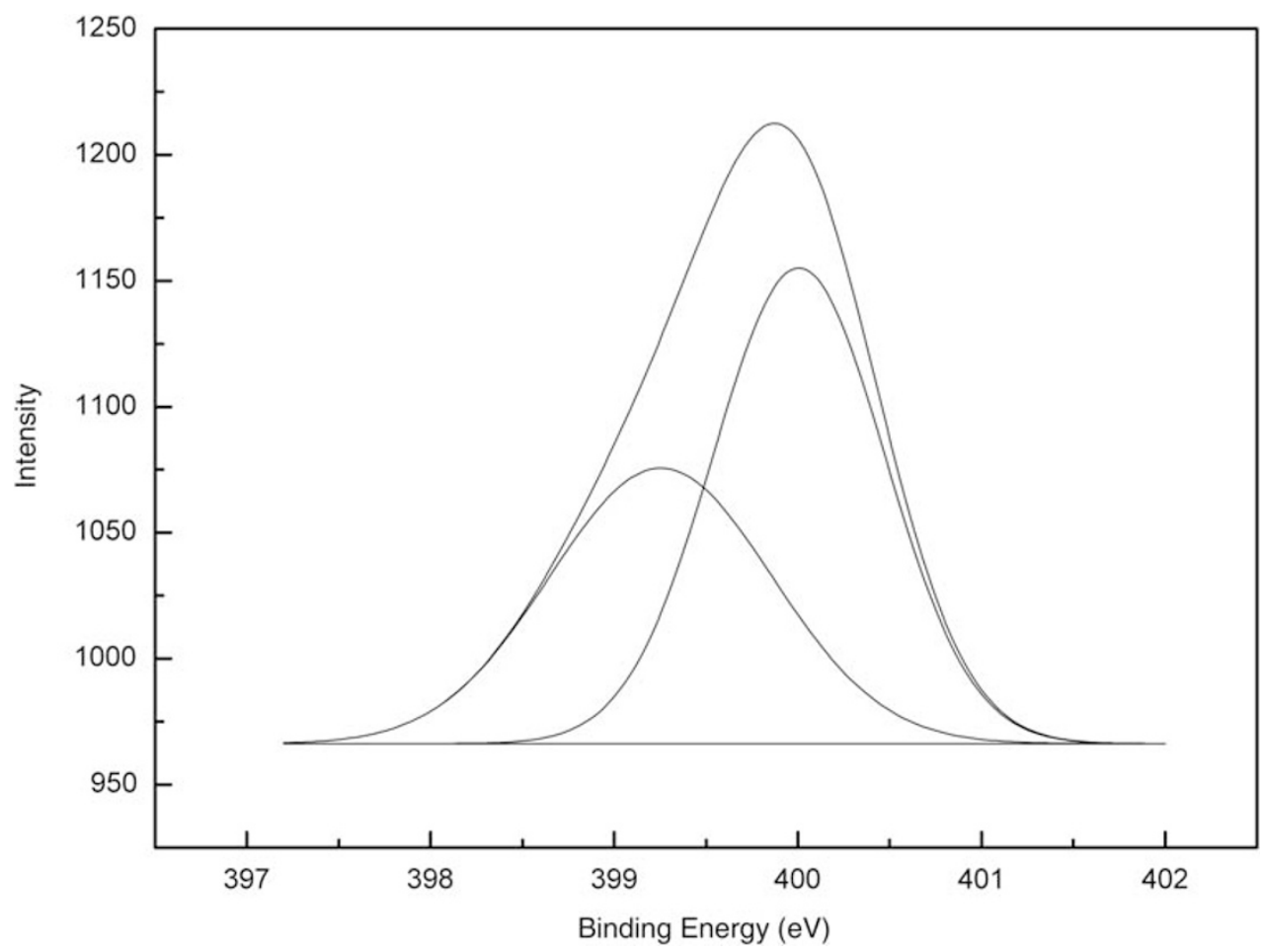

Figure 6 Peak fit of N1s for crosslinked PAM.

$400 \mathrm{eV}$ (188.77), and the later area percentage of the total N1s is about $63 \%$. The samples, with reaction times of 3,6 and $12 \mathrm{~h}$, were investigated by XPS, the N1s spectra of which are illustrated in Figure 7. The peak position of N1s moved toward a higher binding energy step by step with an increase in reaction time, which illustrates that the generation of secondary amines increased during the progres- sion of the reaction. Thus, the results of molecular modeling are confirmed by XPS analysis.

\section{CONCLUSION}

The crosslinking reaction mechanism of PAM/resol has been clarified on the basis of computer simulation and experimental characterization. 


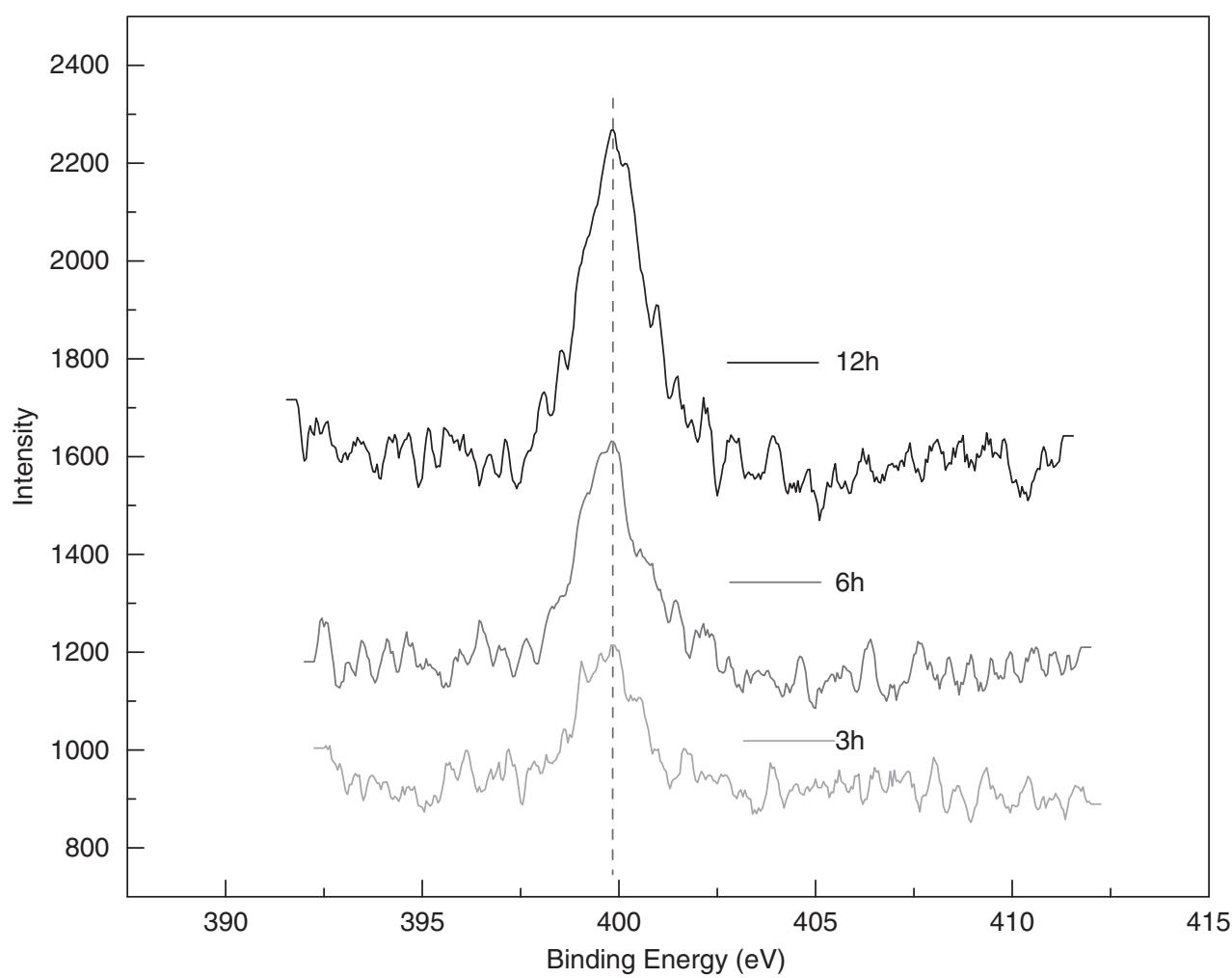

Figure $7 \mathrm{~N}_{1 \mathrm{~s}}$ electron spectra of crosslinked PAM with different reaction times.

The condensations of resol/resol and PAM/resol occurred simultaneously at $353 \mathrm{~K}$ on the basis of thermodynamics, and the energy barrier of the ortho condensation of PAM/resol is the lowest among all crosslinked reactions on the basis of kinetics. The amidocyanogen involved in the crosslinked reaction occupied about $60 \%$ of the total amidocyanogen by simulation and XPS analysis. Thus, the results of molecular modeling and experiments prove that the polycondensation reaction between PAM and resol is the main reaction in the system. The understanding of this mechanism helps the development of new types of PAM gel with good salt and temperature resistance. Experimental characterization combined with molecular simulation afforded a new method to study the crosslinking reaction mechanism.

\section{ACKNOWLEDGEMENTS}

We thank Prof G-S Huang of the State Key Lab of Polymer Materials and Engineering, Sichuan University for her encouragement in commencing the research. The computation of the transition state was accomplished with assistance from Dr XY Li of the Center for Computation Chemistry and Biology Sichuan University. We also thanks the financial assistance provided by Shengli Oilfield Sinopec.

1 Kwon, H. J., Osada, Y. \& Gong, J. P. Polyelectrolyte gels-fundamentals and applications. Poly. J. 38, 1211-1219 (2006).

2 Suematsu, K. Theory of gel formation. Poly. J. 39, 1328-1336 (2007).

3 Bajpai, A. K. \& Giri, A. Water sorption behaviour of highly swelling (carboxy methylcellulose-g-polyacrylamide) hydrogels and release of potassium nitrate as agrochemical. Carb. polym. 53, 271-279 (2003).

4 Akkaya, R. \& Ulusoy, U. Adsorptive features of chitosan entrapped in polyacrylamide hydrogel for $\mathrm{Pb}^{2+}, \mathrm{UO}^{2+}$, and $\mathrm{Th}^{4+}$. J. Haz. Mat. 151, 380-388 (2008).

5 Soppirnatch, S. K. \& Aminabhavi, T. M. Water transport and drug release study from cross-linked polyacrylamide grafted guar gum hydrogel microspheres for the controlled release application. Eur. J. Pharm. Bioph. 53, 87-98 (2003).
6 Vossoughi, S. Profile modification using in situ gelation technology-a review. J. Petr. Sci. Eng. 26, 199-209 (2000).

7 Burrafato, G., Carminati, S., Bonaccorsi, F. \& Lockhart, T. P. Evidence for molecular cross-links in $\mathrm{Cr}^{3+}$ /polyacrylamide gels. Macromolecules. 23, 2402-2406 (1990).

8 Nijenhuis, K., Mensert, A. \& Zitha, P. L. J. Viscoelastic behavior of parly hydrolyzed polyacrylamide/chromium (III) gels. Reh. Acta. 42, 132-141 (2003).

9 Nijenhuis, K. Thermoreversible networks viscoelastic properties and structure of gels. Adv. Polym. Sci. 130, 267 (1997).

10 Grattoni, C. A., Al-Sharji, H. H., Yan, C., Muggeridge, A. H. \& Zimmerman, R. W. Rheology and permeability of crosslinked polyacrylamide gel. J. Coll. Int. Sci. 240, 601-606 (2001).

11 Al-Muntasheri, G. A., Nasr-El-Din, H. A. \& Hussein, I. A. Rrheological investigation of a high temperature organic gel used for water shut-off treatments. J. Petr. Sci. Eng. 59, 73-83 (2007).

12 Zitha, P. L. J., Botemans, C. W., Hoek, J. \& Vermolem, F. J. Control of flow through porous media using polymer gels. J. Appl. phys. 92, 1143-1153 (2002).

13 Shimizu, H., Wakayama, T., Wada, R., Okabe, M. \& Tanaka, F. Solvent effect on junction size in syndiotactic polystyrene physical gel. Poly. J. 37, 294-298 (2005).

14 Takahashi, H., Ishimuro, Y. \& Watanabe, H. Nonlinear mechanical behavior of scarcely crosslinked poly(dimethyl siloxane) gel: effect of strand length polydispersity. Poly. J. 40, 465-474 (2008).

15 Tsuboi, Y., Nishino, M. \& Kitamua, N. Laser-induced reversible volume phase transition of a poly (N-isopropylacrylamide) gel explored by raman microspectroscopy. Poly. J. 40, 367-374 (2008).

16 Ahmad, M. A. A review of thermally stable gels for fluid diversion in petroleum production. J. Petr. Sci. Engin. 26, 1-10 (2000).

17 Gao, P., Zhang, Y. F. \& Huang, G. S. Kinetics study on the formation of resol with high content of hydroxymethyl group. J. Appl. Polym. Sci. 107, 3157-3162 (2008).

18 Delley, B. An all-electron numerical method for solving the local density functional for polyatomic molecules. J. Chem. Phys. 92, 508 (1990).

19 Delley, B. From molecules to solids with the $\mathrm{DMol}^{3}$ approach. J. Chem. Phys. 113, 7756 (2000).

20 Yarovsky, I. \& Evans, E. Computer simulation of structure and properties of crosslinked polymers: application to epoxy resins. Polymer. 43, 963-969 (2002).

21 Bohr, F. \& Henon, E. Comparison of various quantum chemistry methods for the computation of equilibrium constants. J. Phys. Chem. A. 102, 4857-4862 (1998).

22 Chen, Z. J., Lu, X. L. \& Mi, Y. L. Manipulating the surface properties of polyacrylamide with nitrogen plasma. Eur. Poly, 42, 2914-2920 (2006).

23 Allen, G. C., Hallam, K. R. \& Eastman, J. R. XPS analysis of polyacrylamide adsorption to kaolinite XPS analysis of polyacrylamide adsorption to kaolinite quartz and feldspar. Surf. Int. Analy. 26, 518-523 (1998). 